\title{
Cisaillement gravitationnel et sondage de I'Univers
}

Yannick Mellier

Institut d'Astrophysique de Paris et LERMA, CNRS UMR 8112, Observatoire de Paris Lauréat du prix Jean Ricard 2005 de la SFP

\section{L'effet de lentille}

gravitationnelle,

responsable de la

déflexion des rayons

lumineux par la matière,

engendre des effets de

déformation apparente

des objets célestes :

le cisaillement

\section{gravitationnel.}

Les déformations des

galaxies nous révèlent

la distribution de la

matière projetée sur le

ciel. Les cosmologistes

savent aujourd'hui

exploiter les caractéris-

tiques de ces phénomènes

pour reconstruire celles

de l'univers et déterminer

ainsi son contenu en

matière noire et en

énergie sombre.

\section{Les lentilles gravitationnelles}

La relativité générale proposée par Einstein en 1915 est une théorie de la gravitation dont la validité est aujourd'hui confortée par l'accord entre ses prédictions et tous les tests expérimentaux. La gravitation étant une interaction à longue portée, elle constitue la clef de voûte de notre description de l'Univers à grande échelle et sert de cadre général à l'interprétation des observations astronomiques. Une de ses prédictions les plus remarquables est la déflexion des rayons lumineux lorsqu'ils croisent des concentrations de matière au cours de leur propagation. Ce phénomène de "lentille gravitationnelle" illustre qu'en relativité générale les photons suivent des géodésiques d'un espace-temps courbe dont les déformations sont localement déterminées par les concentrations de matière. En modifiant la propagation des photons, les objets astronomiques nous apparaissent déplacés sur le ciel par rapport à leur position réelle, en l'absence de déflexion gravitationnelle (voir figure 1). La mesure de la déflexion de rayons lumineux des étoiles observées près du bord du Soleil lors de l'éclipse de 1919 compte parmilessuccès historiques des prédictions d'Einstein.

C'était aussi une prouesse pour l'époque, car les déflexions gravitationnelles sont en général de très faible amplitude : environ une seconde d'arc pour une masse déflectrice comme le Soleil, moins de dix secondes d'arc pour une galaxie de $10^{11}$ masses solaires, et environ 30 secondes d'arc pour une structure comme un amas de galaxies de $10^{15}$ masses

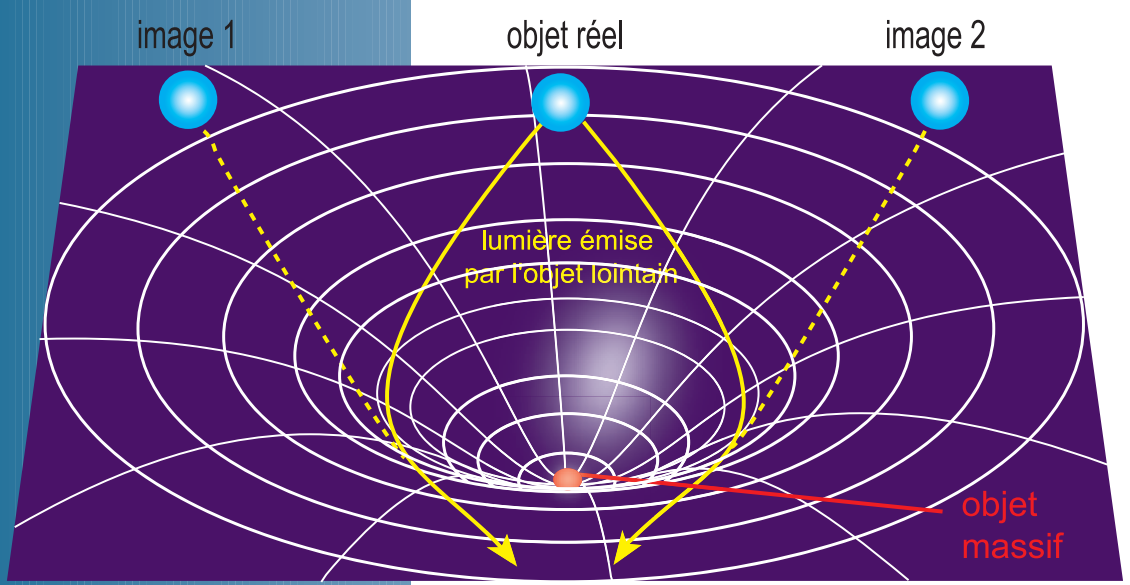

vers la terre solaires. (À titre de comparaison, la taille angulaire de la Lune à la Pleine Lune est de 1800 secondes d'arc environ).

Les lentilles gravitationnelles sont caractérisées par la distance et la position apparente sur le ciel de l'objet défléchi (la source) et du déflecteur (la lentille). Lorsque la masse de la lentille est connue, l'ensemble définit une configuration d'optique gravitationnelle, un banc d'optique cosmique. Pour un objet déflecteur quasiponctuel, comme peut l'être une étoile, l'amplitude de l'angle de déflexion dépend de la masse totale contenue dans la lentille. Si la configuration peut être parfaitement décrite, la mesure de l'angle détermine directement la masse du déflecteur. Comme l'avait perçu l'astronome Fritz Zwicky dès les années 1930, c'est ici que se trouve l'intérêt astrophysique des lentilles gravitationnelles : elles peuvent nous servir de véritable balance cosmique, une sonde à distance permettant de mesurer la masse contenue dans les condensations de matière distribuées dans l'univers. Ce qui est remarquable, c'est que pour mesurer les masses il suffit de connaître les distances de la source et de la lentille ainsi que leurs positions apparentes sur le ciel. À aucun moment, l'état dynamique de la lentille ou les propriétés thermodynamiques des gaz la constituant n'interviennent. C'est un effet très pur, qui est parfaitement décrit par les équations de la relativité générale.

L'intuition de Fritz Zwicky fut confirmée au cours des trente dernières années, après la découverte du premier quasar à image multiple (1979), puis du premier arc gravitationnel (1987). Dans ces deux cas, les astronomes ont découvert des configurations spécifiques de lentilles gravitationnelles reflétant certaines de leurs remarquables propriétés : amplification de la lumière de la source, démultiplication de ses images, altération de sa forme apparente. Dans le cas du quasar multiple, la lentille gravitationnelle était une galaxie, alors que pour le premier arc gravitationnel, il s'agissait d'un amas de galaxies.

Figure 1. Géodésiques suivies par les photons dans un espacetemps courbe. Au centre se trouve une concentration de matière. L'espace-temps est localement altéré par ce champ gravitationnel. Les photons émis par l'objet d'arrière-plan sont défléchis et semblent provenir de deux images différentes. Cette figure illustre à la fois l'effet de déflexion des rayons lumineux et l'effet de démultiplication des images. (Redessiné à partir de la figure S3.19 tirée de "Cosmic perspective", Pearson,Addison Wesley). 


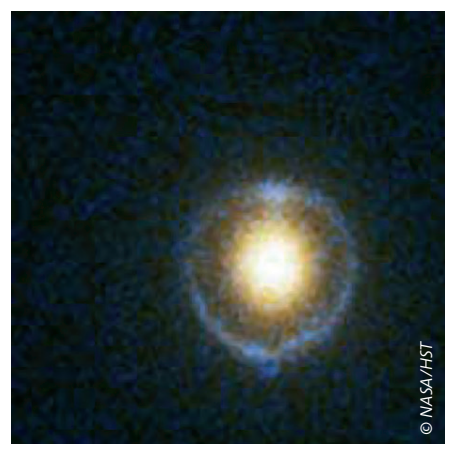

Figure 2. Anneau d'Einstein quasi-parfait détecté par le télescope spatial Hubble. L'objet central est une galaxie et localise la lentille gravitationnelle responsable de la formation de l'anneau.

Figure 3. Une illustration de la multitude des effets de déformation gravitationnelle induits par un seul amas de galaxies: Abell 1689. Les objets les plus étendus et brillants de ces images sont des galaxies appartenant physiquement à l'amas de galaxies. Chaque cadre est un zoom de différents secteurs du même amas de galaxies observé par le télescope spatial Hubble. On y distingue une incroyable quantité d'arcs gravitationnels. La plupart des petits arcs sont des images de sources différentes, les arcs les plus grands étant en général composés de plusieurs images de la même source.
Les configurations de lentille gravitationnelle sont en général un peu plus complexes que celle décrite par une lentille ponctuelle. La distribution de la matière dans la lentille ainsi que la position relative de la source par rapport au centre du déflecteur, sa taille et sa forme intrinsèque interviennent aussi. Lorsque la distance apparente entre la source et le déflecteur croît, l'amplitude de l'effet décroît. Lorsque la source est étendue, la position relative de chacun de ses éléments vis à vis du centre du déflecteur est différente, ce qui induit des déflexions différentielles altérant la forme initiale de la source. Dans une configuration idéale où l'observateur, le déflecteur et la source seraient parfaitement alignés, l'image de la source est transformée en anneau parfait centré sur le déflecteur, c'est un "anneau d'Einstein" (voir figure 2 et encadré page 9). Si l'on déplaçait alors légèrement la source hors de l'axe, la symétrie serait brisée et l'anneau se transformerait en "arc gravitationnel". Ces configurations, qui forment des images astronomiques spectaculaires, ont toutes été observées. Elles constituent des cas d'école qui valident les prédictions théoriques de "l'optique gravitationnelle" et qui fournissent aux astrophysiciens des cas quasi-idéaux de balance cosmique.

\section{Voir la matière invisible}

Si la découverte du premier quasar multiple fut une vérification des prédictions de l'optique gravitationnelle de la relativité générale, les arcs gravitationnels découverts dans les amas de galaxies ont véritablement hissé les lentilles gravitationnelles du rang de phénomène astronomique à celui d'outil d'exploration et de diagnostic pour l'astrophysique extragalactique et la cosmologie. Ils ont montré que, grâce aux déformations gravitationnelles des galaxies lointaines, il devient désormais possible de reconstruire la distribution de masse d'objets astronomiques aussi complexes que les amas de galaxies.

Les amas de galaxies sont des structures gigantesques composées de milliers de galaxies confinées dans un volume de quelques millions d'années-lumière de diamètre et qui baignent dans un gaz ionisé très chaud (quelques centaines de millions de degrés). Ce gaz est révélé par sa forte émission de rayons X. En supposant que ces systèmes sont stationnaires et stabilisés, il est facile de montrer que ce confinement n'est possible que si la masse totale des amas de galaxies atteint $10^{15}$ masses solaires, soit plus de cent fois la masse correspondant aux objets détectés par leur rayonnement électromagnétique, les étoiles ou le gaz. Cet écart considérable signifie que non seulement l'essentiel de la masse des amas de galaxies n'est pas visible, mais aussi qu'il y a dix fois plus de masse dans les amas que dans les galaxies qui le composent, et enfin que cette matière ne peut pas être baryonique (c'est-à-dire être faite de nucléons).

Ces implications sont difficiles à accepter sans au préalable mettre en doute l'interprétation des données elles-mêmes. Peut être que les amas de galaxies ne sont pas stationnaires, que l'état thermodynamique de leurs composantes (galaxies, gaz chaud) est mal compris, ou que la forme générale de leur potentiel gravitationnel est complexe et s'écarte fortement d'un cas idéal, sphérique. Pendant plus de cinquante ans, les astrophysiciens sont donc restés perplexes.

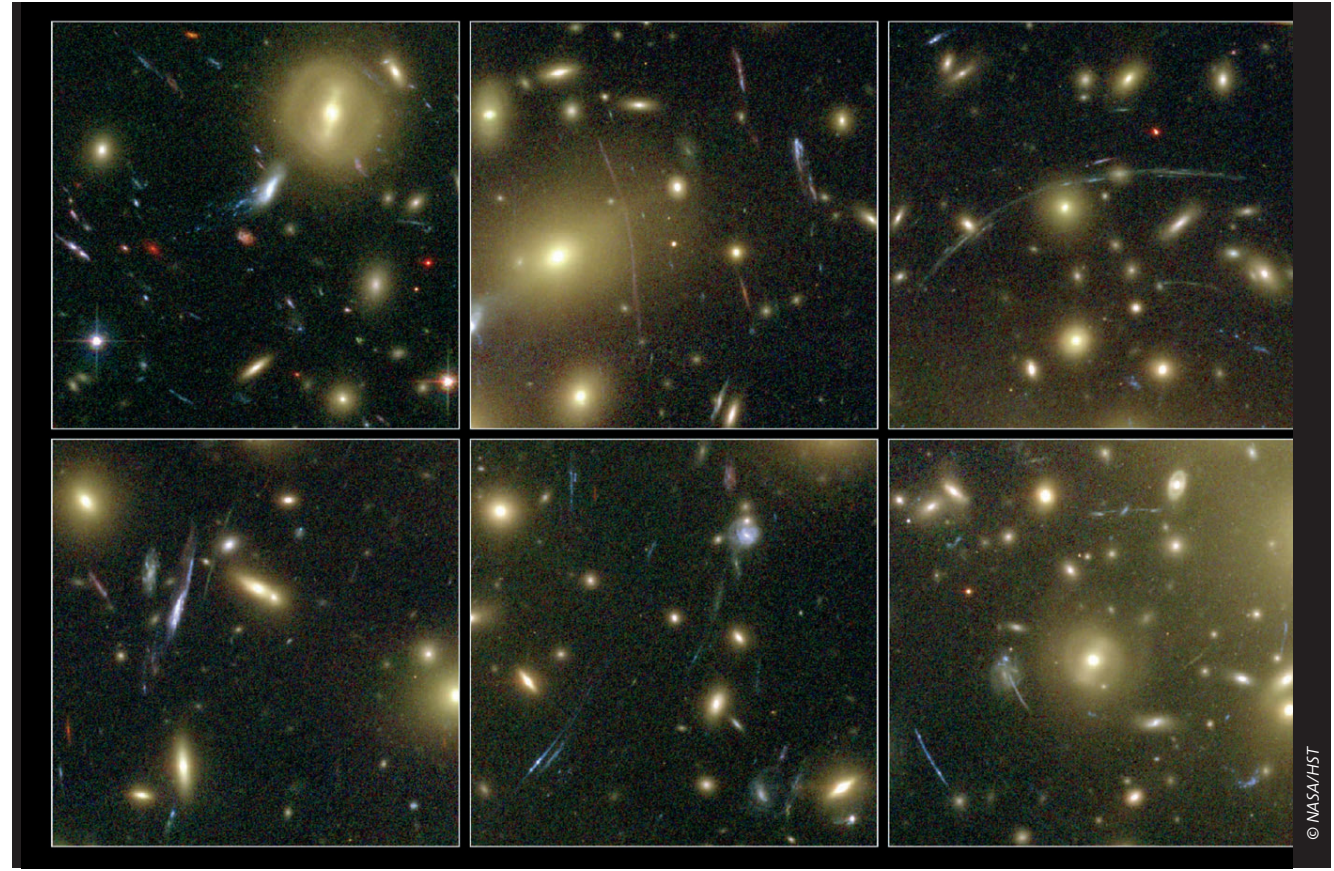


La découverte de l'arc gravitationnel dans l'amas de galaxies Abell 370, il y a exactement 20 ans, a permis de franchir une étape décisive. En effet, puisque le phénomène ne dépend que de la quantité de matière localisée dans le déflecteur, la mesure de la position de l'arc relativement au centre de l'amas de galaxie permet de déterminer directement sa masse. Il a ainsi été possible de vérifier avec une quasi-certitude qu'effectivement les amas de galaxies sont totalement dominés par une matière invisible, dix fois plus abondante que dans les galaxies, et qui maintient le confinement gravitationnel. C'est un résultat particulièrement important et, à l'époque, sans doute la preuve la plus solide que la matière noire doit dominer le contenu matériel de l'Univers et doit être de nature non baryonique.

Plus d'une quarantaine d'amas de galaxies présentant des arcs gravitationnels ont été répertoriés depuis lors. L'optique gravitationnelle appliquée cas par cas confirme systématiquement les conclusions des premières découvertes. Cependant, il y a quelques restrictions limitant leur portée astrophysique. Ces phénomènes sont en effet des cas exceptionnels produits dans des configurations optiques idéales où la source et le déflecteur se trouvent quasi-alignés avec l'observateur. Ce sont donc des événements rares, puisque nous savons que plus de 100000 amas de galaxies sont observables avec les moyens d'observation astronomique actuels. De plus, les arcs gravitationnels nous renseignent précisément, mais uniquement, sur la quantité de matière projetée le long de la ligne de visée et contenue dans un double cône dont la section est dessinée par l'image de l'arc et les sommets sont la galaxie lointaine d'une part et l'observateur d'autre part. Mais fort heureusement, il y a des milliards de galaxies lointaines observables. Dans un angle solide correspondant à la dimension apparente de la Lune, les télescopes actuels peuvent observer plus de $50 \quad 000$ galaxies. Or, un amas de galaxies comme Abell 370 couvre une dimension angulaire d'environ le quart de celle de la Lune. Il y a donc potentiellement plusieurs milliers de galaxies qui subissent, à des degrés divers, le champ gravitationnel de ce déflecteur (voir figure 3).

Sans produire pour chacune un arc spectaculaire, l'amas de galaxies déforme pourtant chacune d'elles d'une amplitude qui dépend d'une part de sa position vis à vis du centre de la lentille et d'autre part de la façon dont la matière est distribuée dans l'amas. Une source de forme circulaire sera ainsi étirée selon une direction bien spécifique, perpendiculaire à la direction du gradient du champ gravitationnel du déflecteur. L'amplitude et l'orientation des déformations des galaxies d'arrière-plan ne sont donc pas aléatoires, et elles sont fortement corrélées. Une analyse statistique permet ainsi de reconstruire le profil de la distribution de masse du déflecteur, en produisant une cartographie des " distorsions gravitationnelles " qui répond à celle de la distribution de la matière noire. Cette matière noire peut donc être "vue ", comme la limaille de fer sur une feuille de papier "voit " le champ magnétique d'un aimant placé au-dessous. La résolution de la cartographie est déterminée par la densité de galaxies d'arrière-plan : plus une observation astronomique est profonde (c'est-à-dire vise des objets lointains), plus la densité de galaxies projetée sur le ciel augmente. Les télescopes actuels atteignent de telles profondeurs que les détails de la distribution de matière dans les amas de galaxies sont identifiés jusqu'aux échelles des galaxies individuelles qui chacune joue le rôle d'une perturbation locale au champ global du déflecteur.

\section{Distorsion gravitationnelle et cosmologie}

Grâce à l'analyse statistique des déformations gravitationnelles, on peut en principe explorer tous les amas de galaxies visibles dans l'univers

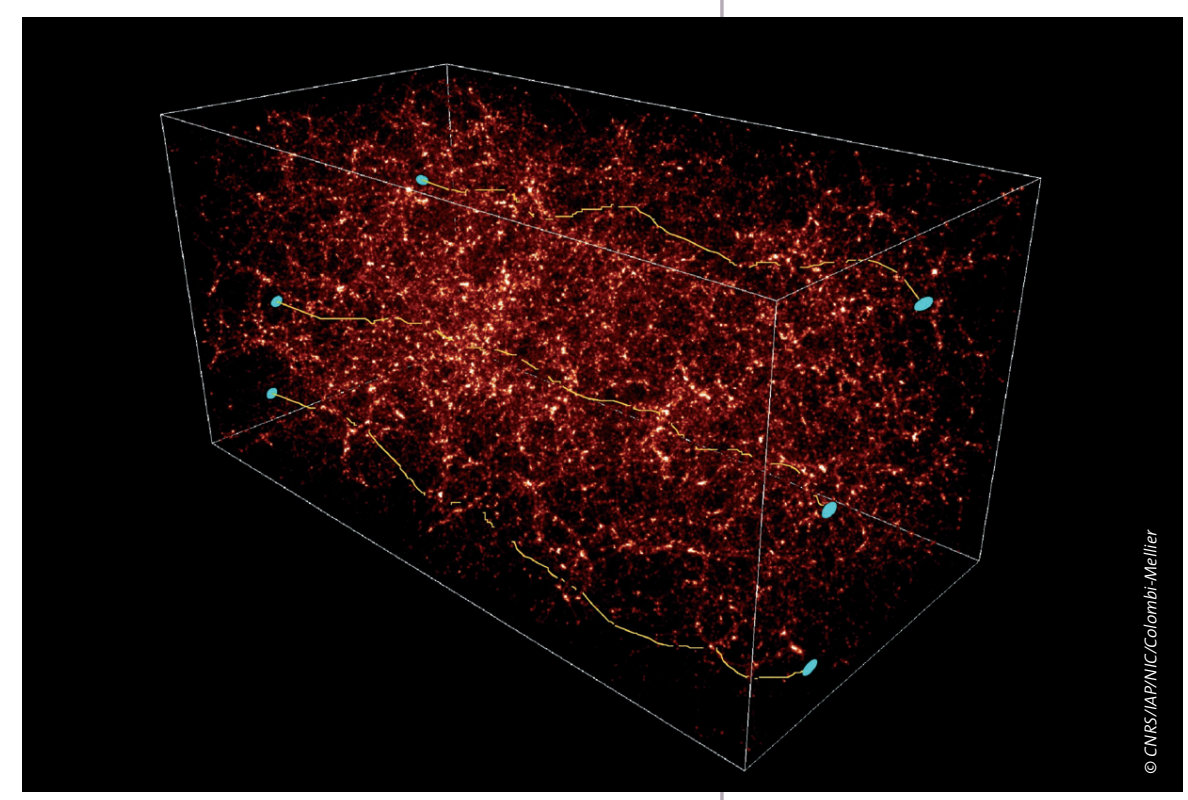

observable et établir des diagnostics statistiques sur ces structures. Cependant, la matière n'est pas uniquement distribuée dans les amas de galaxies. Elle se confine aussi dans des systèmes plus compacts comme des groupes physiques de quelques galaxies, ou dans des structures plus ténues et irrégulières et constituées de dizaines de milliers de galaxies, ou bien de gigantesques filaments dont les dimensions dépassent plusieurs centaines de millions d'années-lumière.
Figure 4. Illustration de la propagation de la lumière dans l'Univers et des déflexions gravitationnelles multiples qu'induit chaque condensation. La boite est une simulation numérique de l'Univers. Sa taille est d'environ 1 milliard d'années lumière de côté.

Au départ, des particules de matière noire sont disposées de façon aléatoire. Puis, des fluctuations croissent et amorcent la formation des structures sous l'effet de la gravitation. L'amplitude et le taux de croissance dépendent du taux d'expansion de l'univers et de son contenu en matièreénergie. Au bout de plusieurs heures de calcul, les simulations numériques restituent ces univers virtuels dont les propriétés sont comparées à celles de l'univers observé.

Les structures rouges et blanches montrent la distribution de matière noire. Plus elles sont claires, plus la structure est dense. On remarque la structuration très filamentaire de l'Univers aux grandes échelles, alors qu'elle semble plus contrastée et compacte à petite échelle. C'est une structuration caractéristique d'un univers dominé par de la matière noire froide. Les lignes jaunes illustrent les géodésiques que suivent les photons et qui sont déformées par les condensations locales de matière. Les ellipses bleues illustrent la forme des galaxies depuis l'émission de la lumière à gauche jusqu'à leur réception sur le télescope à droite. Leur élongation et leur orientation apparente ont été modifiées sous les effets cumulés de toutes les déflexions gravitationnelles. 


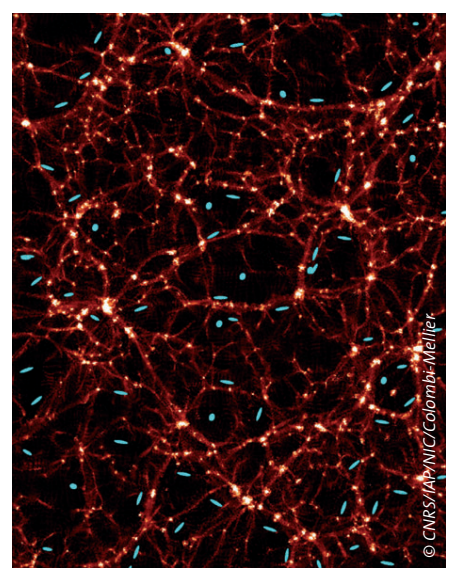

Figure 5. Projection sur le ciel de la simulation montrée sur l'image précédente. La carte montre ce que verrait un observateur avec son télescope: des galaxies dont les ellipticités ne sont plus distribuées aléatoirement, mais dont les orientations et les ellipticités sont corrélées avec la distribution de la matière noire projetée sur le ciel. L'analyse des distorsions gravitationnelles cosmologiques conduite avec le grand relevé CFHTLS permet de reconstruire l'invisible, c'est-à-dire les fluctuations de densité que l'on voit sur cette carte.

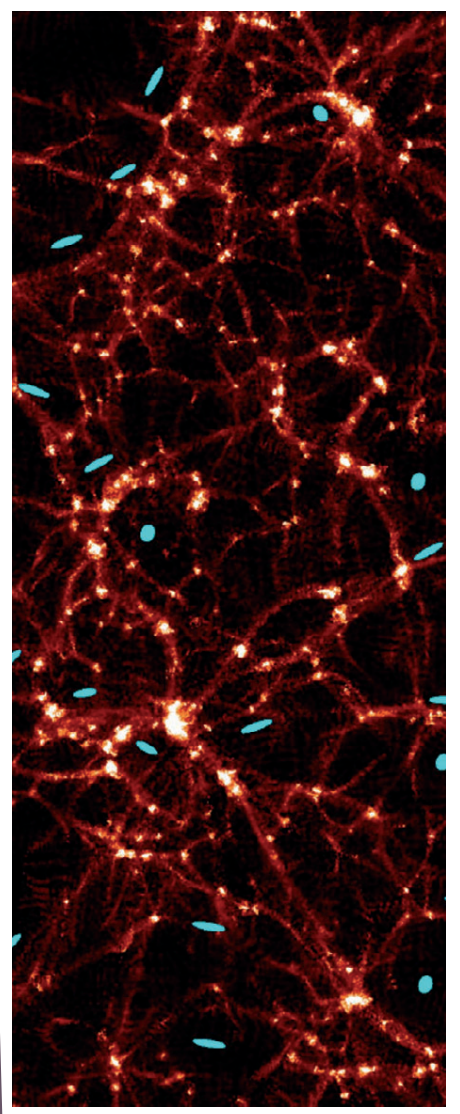

L'ensemble de ces fluctuations de densité couvre un spectre très vaste en dimension et en contraste. Elles résultent de fluctuations initiales qui furent progressivement amplifiées sous l'effet de la gravité au cours de l'histoire de l'expansion de l'Univers. L'histoire de la formation des structures et la structuration actuelle de l'Univers sont déterminées à la fois par son contenu en matière, mais aussi par son contenu en énergie. Or, nous savons grâce aux observations des supernovae que l'Univers ne contient pas uniquement de la matière noire répartie dans les structures mais aussi une composante plus homogène, l'énergie noire, qui est responsable de l'accélération actuelle de son expansion. Tout comme la matière noire, la nature de cette énergie noire est totalement inconnue. On évoque plus singulièrement une constante cosmologique comme étant sa nature probable, mais actuellement on ne peut exclure d'autres hypothèses. L'identification de cette énergie noire est un des enjeux les plus fascinants de la physique fondamentale.

Une des options possibles pour caractériser l'énergie noire est d'adopter une attitude minimaliste, même si elle est incomplète, en supposant que cette composante se comporte comme un fluide parfait. Comme tout indique que notre univers est homogène et isotrope à très grande échelle, cette hypothèse est somme toute plutôt raisonnable. Dans ce cas, l'équation d'état reliant sa pression $\mathrm{P}$ à sa densité d'énergie $\rho$ peut s'écrire sous la forme $\mathrm{P}=\mathrm{w} \rho$, comme celle de la matière noire ordinaire $(\mathrm{w}=0)$ ou celle des photons ( $w=1 / 3)$. Pour l'énergie noire, l'éventail des valeurs de ce paramètre est assez vaste et il pourrait même évoluer au cours du temps. S'il s'agit cependant d'une constante cosmologique, alors $\mathrm{w}$ vaut -1 et est constant. S'il s'agissait par contre d'une composante de type cordes cosmiques, alors on aurait $\mathrm{w}=-1 / 3$. Dans tous les cas, la distribution des fluctuations de densité, caractérisée par le champ de distorsion gravitationnelle, sera différente suivant la valeur de w.

Tout comme la matière noire, certaines de ces propriétés peuvent donc être décryptées au travers du spectre de fluctuations tracé directement par les effets de distorsion gravitationnelle. Mais contrairement à l'étude d'un amas de galaxies, il faut cette fois analyser les déformations gravitationnelles accumulées par toutes les structures traversées le long d'une ligne de visée, puis conduire l'analyse sur une très vaste partie du ciel pour explorer les fluctuations à chaque échelle et reconstruire le spectre complet de densité. Une fois recomposé, il est alors comparé aux prédictions théoriques d'une panoplie de scenarii cosmologiques.
La grande force de l'approche est profondément liée au fait que l'effet de lentille gravitationnelle s'applique à toutes déformations de l'espace courbe, indépendamment de la nature de la lentille ou de sa taille. La cartographie à grande échelle des déformations gravitationnelles peut donc être directement interprétée comme une vision du spectre de fluctuations.

\section{L'observation des distorsions gravitationnelles cosmologiques}

La mesure des distorsions gravitationnelles sur des échelles cosmologiques présente quelques difficultés techniques. Elle demande une analyse précise des formes des galaxies, car les déformations induites par les structures sont de plus en plus faibles lorsque leur dimension augmente. Or les formes observées par les télescopes sont aussi altérées par la turbulence et la réfraction atmosphériques, puis par les aberrations optiques des instruments. L'amplitude de ces contaminations dépasse d'un ordre de grandeur les effets gravitationnels et leur correction est particulièrement difficile. De plus, pour explorer un vaste domaine d'échelle, il faut aussi observer des millions de galaxies sur d'immenses étendues du ciel.

Pour atteindre ces objectifs, un imposant projet est en cours sur le Télescope CanadaFrance-Hawaii (CFHT). Il a commencé par une phase préliminaire entre 1999 et 2003 au cours de laquelle une petite portion du ciel $\left(10 \mathrm{deg}^{2}\right)$ fut observée en détail, au CFHT (le projet VIRMOS-DESCART) et au Very Large Telescope VLT de l'European Southern Observatory (ESO). Ces données ont permis de détecter pour la première fois les effets de distorsions gravitationnelles cosmologiques. Le principe de la méthode, ainsi que les outils d'analyse et d'interprétation, jusqu'à la mesure du spectre de fluctuations de densité, ont ainsi pu être validés. L'amplitude et la forme du signal étaient exactement celles prédites par le modèle cosmologique où les structures se forment suite à des instabilités conduisant à leur effondrement sous l'effet de l'accrétion gravitationnelle.

Mais c'est avec le grand relevé CanadaFrance-Hawaii Legacy Survey (CFHTLS) que le projet prend toute sa dimension. En 2002, le CNRS et le CNRC canadien ont joint leurs efforts pour accorder 500 nuits d'observation au CFHT à ce formidable programme. Elles ont tout d'abord financé un programme de rénovation du foyer primaire du télescope (MegaPrime), et le CEA/DAPNIA a mis au point la plus grande caméra CCD panoramique au monde (MegaCam). Cette caméra est composée 


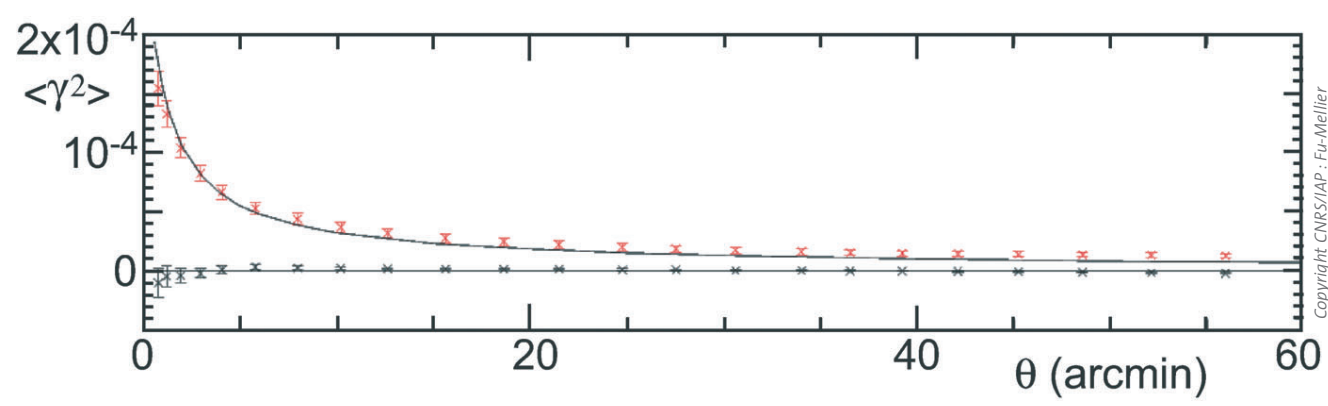

d'une mosaique de 36 CCDs produisant des images de $18000 \times 18000$ pixels et couvrant à chaque pose un champ grand comme quatre fois celui de la Pleine Lune. Les observations ont commencé en 2003. Les images produites par le CFHT sont ensuite traitées au centre de Traitement Terapix localisé à l'Institut d'Astrophysique de Paris. L'ensemble du projet porte sur plus de 100 Tera-octets d'images accumulées au cours des années 2003 à 2008. Un grand programme franco-canadien d'analyse du cisaillement gravitationnel cosmologique a été mis en place pour couvrir $170 \mathrm{deg}^{2}$ du ciel. Il est opérationnel et les premières images accumulées au cours de la période 2003-2004, portant sur environ 15\% des données, ont déjà permis de mesurer le spectre de fluctuations de densité avec une qualité et une précision supérieures à toutes les analyses précédentes (voir figure 6).
Le spectre mesuré a été comparé aux scenarii cosmologiques et montre une remarquable similitude avec celui prédit pour un univers dominé par de la matière noire froide où les structures se forment par effondrement gravitationnel et, plus remarquable encore, où l'énergie du vide aurait des propriétés proches de celles d'une constante cosmologique. Les analyses détaillées avec les données les plus récentes sont en cours et devraient s'achever fin 2008 avec la reconstruction du spectre de fluctuations de densité, depuis les échelles galactiques jusqu'à celles des grands filaments. A partir de ce sondage, il sera alors possible de construire une carte de la matière noire projetée sur le ciel, comme il existe aujourd'hui des cartes montrant la distribution des galaxies, et d'y mesurer les propriétés de la matière noire et celles de l'énergie noire avec une précision de 5 à $10 \%$.

Figure 6. Mesure du cisaillement gravitationnel cosmologique obtenue avec le grand relevé CFHTLS. En abscisse sont portées les échelles angulaires, en ordonnée l'amplitude de l'excès d'ellipticité des galaxies induit par les effets de lentille gravitationnelle. Les points rouges sont les résultats des mesures. Les points noirs sont les résidus d'effets systématiques. La courbe noire est la prédiction du scénario cosmologique avec la valeur de la constante cosmologique déterminée grâce aux observations des supernovae. II ne s'agit pas d'un ajustement, mais simplement d'une superposition. La compatibilité est saisissante. On imagine la précision espérée à la fin du relevé, alors que cette figure est construite avec $15 \%$ des données seulement.

\section{Bibliographie}

A. Bouquet et E. Monnier,

"Matière noire et autres cachotteries de l'Univers",

Dunod, collection Quai des Sciences, Paris (2003).

\section{G. Soucail et al,}

(découverte du premier arc gravitationnel), Astronomy \& Astrophysics, 172 (1987), L14.

D. Walsh et al, (découverte $d u$ premier quasar à arc multiple), Nature, 279 (1979), 381.

F. Bernardeau, "Les mirages de la gravitation ", Pour la science, 326 (2004), 84.

En relativité générale, une masse ponctuelle $M$ défléchit un rayon lumineux - ou toute autre onde électromagnétique - passant à une distance $d$ par un angle $\alpha=4 G M / c^{2} d$, et ceci indépendamment de la longueur d'onde du rayonnement (ici, G est la constante de gravitation de Newton et c la vitesse de la lumière).

Dans le cas idéal d'un amas de galaxies dont la distribution de masse est à symétrie sphérique, si le centre de celui-ci est aligné sur la droite joignant la galaxie-source à l'observateur terrestre, l'image vue est un anneau (voir figure) dont le rayon angulaire vaut :

$\mathrm{e}=\left[\left(4 G M / C^{2}\right) \cdot(1-x) / D x\right]^{1 / 2}$, où D est la distance de l'observateur à la source, $D x$ celle à la lentille et M la masse de la lentille. Ce cas a été calculé par Einstein dès 1912, mais il ne le publia qu'en 1936. Entre temps, le phénomène avait également été décrit séparément par Chwolson et Eddington vers 1920. Sa première observation est bien plus récente (années 1990).

Si l'on est capable de mesurer les distances D et Dx (par l'intermédiaire du décalage spectral de la source et de la lentille), la mesure du rayon de l'anneau fournit ainsi la masse M. Par exemple, pour une source très lointaine $(x<1)$ et un amas situé à 200 mégaparsecs ( 650 millions d'années lumière), un rayon de l'anneau de 20 secondes d'arc correspond à une masse de $10^{13}$ fois celle du Soleil.

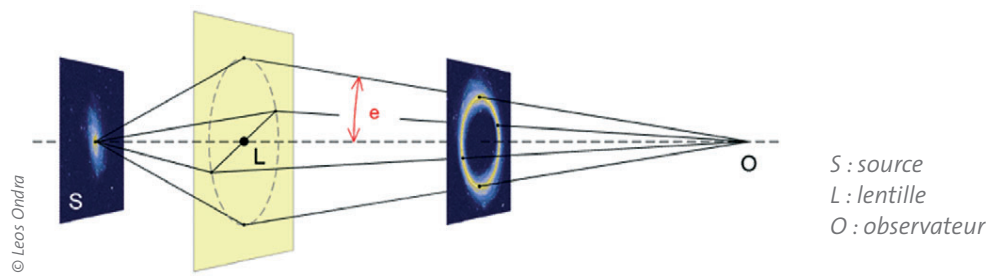

F. Bernardeau, Y. Mellier,

"Les distorsions gravitationnelles pour cartographier l'univers", Images de la physique 2003-2004, Editions du CNRS, Paris (2004), pp. 42-51.

\section{Y. Mellier,}

"Probing the Universe with Weak Lensing ", Annual Review of Astronomy and Astrophysics, 37 (1999) 127.

A. Refrégier, "Weak gravitational lensing by large-scale structure ", Annual Review of Astronomy and Astrophysics, 41 (2003) 645

L. van Waerbeke, Y. Mellier "Cosmic Shear on distant Galaxies", dans "Impact of gravitational Lensing on Cosmology ",

IAU Symposium 225, Y. Mellier et G. Meylan eds, Cambridge University Press (2005). 